\title{
Individual blade pitch control of floating offshore wind turbines for load mitigation and power regulation
}

\author{
Saptarshi Sarkar, Breiffni Fitzgerald, and Biswajit Basu, Member, IEEE
}

\begin{abstract}
This paper proposes a new strategy for individual blade pitch control to regulate power production while simultaneously alleviating structural loads on spar-type floating offshore wind turbines. Individual blade pitch control types of algorithms for offshore wind turbines are sparse in the literature though there are expected benefits from experience on such types of controllers for onshore wind turbines. Wind turbine blade pitch actuators are primarily used to maintain rated power production at above-rated wind speeds and therefore, control algorithms are usually developed only to regulate power production. The scope of reducing structural loads using individual pitch control has been proved to be very promising over the last decade and numerous individual pitch control algorithms have been proposed by researchers. However, reduction in structural loads often results in a degradation in power production and regulation. Furthermore, improving power regulation often has a detrimental effect on the floating platform motion. In this paper, a new control strategy is proposed to achieve the two competing objectives. The proposed controller combines a low authority Linear Quadratic (LQ) controller with an integral action to reduce the 1P (once per revolution) aerodynamic loads while regulating power production using the same pitch actuators that are traditionally used only to optimize power production. The proposed controller is compared against the baseline controller used by state-of-the-art wind turbine simulator FAST using a high fidelity aeroelastic offshore wind turbine model. Numerical results show that the proposed controller offers improved performance in optimizing power production and reducing wind turbine and platform loads compared to the baseline controller over an envelope of windwave loading environment.
\end{abstract}

Index Terms-Floating offshore wind turbines, individual blade pitch control, regulate power production, alleviate aerodynamic loads.

\section{INTRODUCTION}

W ITH the increasing demand for renewable energy, offshore wind energy is set to become one of the major sources of renewable energy in years to come. Hence, it is important to optimize the performance of these expensive structures to increase/improve their energy production and design life span. Offshore wind turbines are subjected to a stochastic wind-wave environment and the majority of the structural loads are transferred from the blades down to the platform. The rotor of a wind turbine is designed to extract maximum possible mechanical energy from the inflowing wind. In this process the blades are actively pitched to the

The authors are with the School of Engineering, Trinity College Dublin, Dublin, Ireland e-mail: (sarkars@tcd.ie; breiffni.fitzgerald@tcd.ie; basub@tcd.ie).

Manuscript received... inflow wind to maintain the rated rotor speed at above rated wind speeds. While the turbine blades were primarily pitched to maintain rated rotor speed, modern wind turbines also use active pitch control to reduce aerodynamic loads the wind turbines. Some of the earliest works on individual pitch control was presented in Bossayni [1], [2]. The developed individual pitch control strategies were further verified by field experiments in [3].

Since then, numerous individual pitch control strategies have been proposed by researchers with different control objectives. A discussion on various pitch control strategies from basic PID (proportional-derivative-integral) controllers to complex multivariable controllers like $H_{\infty}$, neural network, adaptive control etc., has been presented in [4]. The study also focused on variable-speed-variable-pitch-control, an emerging trend in wind turbine technology and concluded that control of this nature is complex due to the highly non-linear nature of the wind turbine. An innovative $\ell_{1}$-control scheme using two decoupled linear time-invariant models derived using Colemen Transformation to design individual and collective pitch controllers independently was proposed in [5]. The individual pitch controller was designed to reduce blade root bending moments and the collective pitch controller was designed to maintain rated rotor speed. The authors reported significant load reduction without loss in energy production for onshore wind turbines. A quite similar work was presented by [6] where a gain scheduled $\ell_{1}$-optimal control strategy for variable-speedvariable-pitch wind turbines was proposed. The controller was developed using linear models of the wind turbine at different operating points using genetic algorithm optimization. The controller was coupled to the wind turbine simulator FAST [7] and compared against a well tuned PI (proportional-integral) controller. The authors reported improved power quality and decrease in generator torque and rotor speed fluctuations.

A disturbance accommodating controller (DAC) was proposed in [8] with the aim to extend fatigue life by reducing mechanical loads on the wind turbine while maintaining the rated rotor speed. FAST [7] was used as the simulation tool and the wind turbine was subjected to stochastic wind loads. The controller was shown to perform better than the conventional collective or individual pitch controller.

Model Predictive Control has gain popularity since 1980's in industrial application and the same has been proposed for wind turbine control. LIDAR measurements can provide information about wind at various distances in-front of the wind turbine. This information was used to design a nonlinear model pre- 
dictive controller in [9]. The controller was compared against the baseline controller and was shown to reduce extreme gust loads by $50 \%$ and lifetime fatigue loads by $30 \%$. A nonlinear model predictive controller for floating offshore wind turbines has been proposed in [10].

Petrovic et al. [11] discussed the development of a control algorithm to mitigate higher order dynamics arising from rotor asymmetries. The paper showed that rotor asymmetries introduce additional oscillations of both structural loads and rotor speed and standard control algorithms for reduction of structural loads cannot reduce them. The existing control algorithm for reduction of structural loads was augmented to enable reduction of structural loads caused by rotor asymmetries.

Gao and Gao [12] developed an optimal PI controller by optimizing the tuning parameters using a direct search optimization algorithm. The authors investigated the effect of time delay caused by the hydraulic pressure units and reported that the proposed controller had desired performance against time delays without any need of prior information about the induced delay. Ren et al. [13] proposed a nonlinear PI controller that eliminates the need of switching the controller gains in a gain scheduled PI controller. Hence, it requires only one set of PI parameters for entire operational region. Simulations showed that the proposed nonlinear PI controller is better than the conventional and gain scheduled PI controllers in regulating constant power and reducing drive-train stresses.

Adaptive control was developed by [14] with disturbance rejection and load mitigation capabilities for onshore wind turbines. It was shown that the adaptive controller was better in load mitigation than the baseline controller employed by FAST [7] but, at a cost of degradation in power regulation. Further, it was also shown that the GSPI (gain scheduled PI) was sensitive to model uncertainties while the adaptive controller was capable of dealing with uncertainties. A fault tolerant controller (FTC) was proposed by [15] that included a adaptive sliding mode observer and a baseline PI controller to estimate parametric pitch actuator faults. It was shown that the proposed FTC is able to maintain stability of wind turbines in the presence of actuator faults. The objective of the controller was to recover nominal baseline PI controller performance under actuator faults. The controller was not developed to reduce structural loads. A wavelet-LQR based individual blade pitch controller was proposed in [16] capable of reducing aerodynamic loads on onshore wind turbines. But again, allowing for degradation in energy production.

Namik and Stol [17], [18] proposed an individual pitch State Feeback Controller (SFC) and Disturbance Accomodating Controller (DAC) for floating offshore wind turbines. In [19] the authors demonstrated the performance of the above two controllers on a spar-buoy floating wind turbine. The authors showed that while both controllers are capable of improving power regulation, the DAC has a detrimental effect on the platform motion. The SFC was inferior compared to the DAC in power regulation, but, the platform rolling and pitching rate was similar to that of the baseline controller. The authors recommended the use of the SFC since the platform motion was not amplified. To summarize, the controller were not capable of improving power regulation and reducing platform motion simultaneously.

The above review summaries some of the key individual pitch controllers proposed in literature that utilize innovative technology and/or complex control algorithms to optimize performance of wind turbines. It has been reported by many researchers that structural load reduction using individual pitch controllers can lead to degradation in output power quality and turbine regulation [14], [16]. The available literature on individual pitch controllers for floating offshore wind turbines (FOWTs) is rather sparse. FOWTs present additional challenges in the design of an appropriate controller as stability of the FOWT (pitching and rolling of the platform) is an important aspect that must be catered for. The IPCs proposed in the literature are not capable of improving platform response and some controllers even have detrimental effects on the platform motion [19]. Thus, it is an open challenge to develop control algorithms that are not only capable of improving power production but are also capable of simultaneously reducing wind turbine and platform loads. This gap is addressed in this paper by proposing a new dual-objective individual pitch control strategy that is both simple and intuitive. The control strategy combines the benefits of a LQ controller with an integral controller to achieve the desired objectives. Unlike the IPCs available in literature the proposed controller is capable of simultaneously reducing rotor speed variability thus improving power production and alleviating structural loads both on the wind turbine and the floating platform and thus increasing life-span and improving stability of the spartype FOWT. A dual-objective control strategy was presented by [20] where two controller, active tendon control and passive blade pitch control were used to decouple vibration control from optimal power control. A Pareto-optimal optimization formulation was used to the controller. However, it must be noted here that the controller proposed in this paper uses only active pitch control used achieve the two competitive objectives.

\section{Proposed Control Strategy}

A non-linear aeroelastic model of the FOWT [21] has been used in this study to simulate its dynamic behaviour subjected to a stochastic wind-wave loading environment and evaluate the performance of the controller. The full non-linear FOWT model has 22 degrees of freedom as listed in Appendix A. Aerodynamic and hydrodynamic loads on the wind turbine are estimated using the Blade Element Momentum (BEM) theory and Morison's equation respectively. The mooring cables are modelled using MoorDyn [22]. For brevity, details on the dynamic modelling of the 22 dof system are not presented in this paper. The reader will find more details on the modelling in [23], [24].

The proposed controller combines a low authority LQ controller and an integral controller to determine the individual blade pitch angles. Although the performance of the controller is evaluated using a fully non-linear model, a reduced order model is used to develop the linear quadratic (LQ) controller. 




Fig. 1. Schematic diagram of the proposed controller

The control input of the individual blade pitch angles are obtained as

$$
\boldsymbol{\Theta}=\theta+\boldsymbol{\Theta}_{l}
$$

where $\theta \in \Re$ is the collective pitch angle obtained from the integral controller and $\Theta_{l} \in \Re^{3 \times 1}$ is the individual pitch angles obtained from a low authority LQ controller. Conceptually, the integral controller is designed to determine the low frequency variation in the pitch angle following the low frequency variation in the inflow wind speed and the LQ controller mitigates the 1P frequency of the aerodynamics loads. The along-wind (prevalent direction of the inflow wind) components of the wind turbine are subjected to the majority of the aerodynamic loads and therefore the chosen degrees of freedom for the reduced order model are

$$
\mathbf{q}=\left\{\begin{array}{llllll}
q_{P} & q_{T F A 1} & q_{B 1 F 1} & q_{B 2 F 1} & q_{B 3 F 1} & q_{\varepsilon}
\end{array}\right\}^{T}
$$

All the degrees of freedom are described in Appendix A except $q_{\varepsilon}$ which is the generator speed error degree of freedom. More details on this degree of freedom is provided in Appendix B. The governing equations of motion are obtained using Kane's method [25]. The resulting non-linear equations of motion are further linearized by ignoring the quadratic and higher order terms and the final set of linearized equations are presented in Appendix B. These equations of motion are time-varying in nature. But, since the time-varying nature in the along wind direction is relatively small in magnitude, the time varying terms are further dropped assuming a frozen rotor situation. The final linear-time-invariant equations can be written in matrix form as

$$
\mathbf{M} \ddot{\mathbf{q}}+\mathbf{C} \dot{\mathbf{q}}+\mathbf{K q}=\mathbf{F}_{\text {Aero }}(v, \boldsymbol{\Theta})+\mathbf{F}_{\text {Hydro }}\left(H_{s}, T_{p}\right)
$$

In the above equation, aerodynamic loads $\mathbf{F}_{\text {Aero }}(v, \boldsymbol{\Theta})$ is a function of wind speed $v$ and blade pitch angles $\boldsymbol{\Theta}$. The hydrodynamic loads $\mathbf{F}_{H y d r o}$ is a function of significant wave height $H_{s}$ (the average height of the highest one-third of all measured waves) and peak spectral period $T_{p}$ (the wave period associated with the most energetic waves in the total wave spectrum at a specific point). The second order equations of motion can be then rewritten in first order form as

$$
\dot{\mathbf{x}}=\mathbf{A x}+\mathbf{B}(\boldsymbol{\Theta}) \boldsymbol{\Theta}+\mathbf{G}\left(v, \boldsymbol{\Theta}, H_{s}, T_{p}\right)
$$

Here, $\mathbf{x}=[\mathbf{q} ; \dot{\mathbf{q}}] \in \Re^{12 \times 1}$ is the state vector, the vector of individual blade pitch angles $\Theta \in \Re^{3 \times 1}$ is the control input. Ideally, the control influence matrix $\mathbf{B}(\Theta)$ is a function of the individual pitch angles. However, to simplify the controller design it is assumed that the controller gain matrix derived around the collective pitch angle $\theta$ and is valid for $\Theta$ (i.e., $\mathbf{B}(\boldsymbol{\Theta}) \approx \mathbf{B}(\theta)$ ). The control influence matrix is obtained from a Taylor series expansion of the aerodynamic loads around the collective pitch angle as the operating point.

$$
\begin{aligned}
\mathbf{F}_{\text {Aero }}(v, \theta) & =\mathbf{F}_{\text {Aero }}\left(v_{0}, \theta_{0}\right)+\left.\frac{\partial \mathbf{F}_{\text {Aero }}}{\partial \theta}\right|_{\left(v_{0}, \theta_{0}\right)} \theta \\
& +\left.\frac{\partial \mathbf{F}_{\text {Aero }}}{\partial v}\right|_{\left(v_{0}, \theta_{0}\right)} v+\mathcal{O}
\end{aligned}
$$

Where $\mathcal{O}$ represents the higher order terms. The control influence matrix can be obtained as

$$
\mathbf{B}(\theta)=\left.\frac{\partial \mathbf{F}_{\text {Aero }}}{\partial \theta}\right|_{\left(v_{0}, \theta_{0}\right)}
$$

The above linearization is performed using a numerical central difference scheme on the complete non-linear model to obtain the control influence matrix using collective pitch control strategy (i.e., equal pitch angles for three blades). Using the above linearized system matrices the steady-state (infinite-horizon) LQ controller is designed ignoring the external disturbance term in equation (4). Although a steady-state LQ controller is not optimal for a finite time system, this approach is a common practice [17], [19] as it renders the design of the LQ controller simpler without the need for solving another set of differential equations for the controller. The cost function for the low-authority LQ regulator can be given as

$$
J=\int_{0}^{\infty}\left(\mathbf{x}^{T} \mathbf{Q} \mathbf{x}+\mathbf{\Theta}_{l}^{T} \mathbf{R} \Theta_{l}\right) \mathrm{d} t
$$

where $\mathbf{Q}$ is state weight matrix that penalizes the control system states and $\mathbf{R}$ is the input weight matrix that penalizes the control input vector. For a steady-state LQ regulator the control input can be obtained from the solution of the Algebraic Ricatti Equation as

$$
\begin{aligned}
& \mathbf{P A}+\mathbf{A}^{T} \mathbf{P}-\mathbf{P B}(\theta) \mathbf{R}^{-1} \mathbf{B}^{T} \mathbf{P}+\mathbf{Q}=\mathbf{0} \\
& \boldsymbol{\Theta}_{l}=-\mathbf{R}^{-1} \mathbf{B}(\theta)^{T} \mathbf{P} \mathbf{x}(t)=-\mathbf{K}(\theta) \mathbf{x}(t)
\end{aligned}
$$

A schematic diagram of the controller is shown in Figure 1. The LQ controller is combined with an integral action as shown in Figure 1. The integral controller has been obtained from [26], [27]. It has been pointed out in [26], [27] that the controller response frequency must be lower that the smallest response frequency of the FOWT and the wave loading frequency. This must be ensured so that the response of the FOWT is not amplified by the controller. Hence, the integral gains $K_{I}$ are chosen to attain a controller-response natural frequency of $0.2 \mathrm{rad} / \mathrm{s}$. A controller response frequency of $0.2 \mathrm{rad} / \mathrm{s}$ is lower than the platform pitch natural frequency and wave loading frequency in most sea-states and hence the controller remains positively damped. The final control input is obtained from equation (1). 
To summarize the proposed controller, the LQ controller is designed to mitigate the $1 \mathrm{P}$ vibrations of the blades, the tower fore-aft vibrations and the platform pitch motion. The integral action guarantees convergence towards the rated speed in the above rated wind region while the LQ controller optimizes the dynamic characteristics of the responses. The combined action of the two controllers have proved to be very effective in optimizing the power production of a FOWT and reducing structural loads as will be shown in the following sections. It can also be observed from Figure 1, that the measurements are first passed through a low-pass filter to eliminate the high frequency content and the control input is saturated prior to feedback. The saturation limits are prescribed in [24] to deliver realistic control actions to the blade pitch actuators.

\section{Controller Performance - Steady Wind}

In this section the performance of proposed controller is compared against the baseline controller when the FOWT is subjected to steady wind and no wave loads. The main purpose of this section is to study the response of the FOWT and to evaluate whether the proposed IPC is capable of improving the transient behavior of the FOWT. The 5MW OC3 Hywind turbine, a spar-type FOWT, defined in [27] has been used for numerical purposes. The structural and aerodynamic properties of the tower and the blades are defined in [26]. MATLAB [28] has been used as the simulation platform. A sampling rate of $40 \mathrm{~Hz}$ has been used for time integration using the rungakutta 4th order method. To investigate the transient behavior the FOWT is subjected to a steady wind field with vertical shear. The hub height reference wind speed is $18 \mathrm{~m} / \mathrm{s}$ and it is assumed that it is floating on still water. As mentioned in the previous section the integral gain $K_{I}$ is obtained from [27]. Therefore only the LQ controller needs to be designed by proper selection of the state weight matrix and the input weight matrix. For ease of design the control weight matrix is chosen as $\mathbf{R}=\mathbf{I}_{3 \times 3}$.

It is important to note here that we are aiming for a low authority controller. Unlike conventional mechanical systems, the pitch actuators cannot be used to drive the error state to zero. Designing a high authority controller will demand high pitch actuation which is not suitable for a wind turbine. Pitching of the blades will also be limited by its saturation limits. The aim is to design a low authority controller that is capable of reducing the aerodynamic loads with relatively lower pitch actuation. Therefore, the state weight matrix is chosen as $\mathbf{Q}=0.001 \times \mathbf{I}_{12 \times 12}$. The state weight matrix $\mathbf{Q}$ is further modified by increasing the weights on the tower foreaft, platform pitch and generator azimuth states to 1 to obtain the final low authority controller. It must be noted here that the selected controller weight matrices are used over the entire wind-wave loading environment without the need to retune them with changing wind speed [6].

The numerical time history predictions are shown in Figure 2. The baseline controller (BC) is compared against the proposed IPC with and without the integral controller (IPC-W/ Integral Controller, and, IPC-W/O Integral Controller, respectively). Figures $2 \mathrm{a}$ through $2 \mathrm{f}$ show the structural response of the FOWT. Figure $2 \mathrm{~g}$ shows the rotor speed which should track the rated speed of $12.1 \mathrm{rpm}$ to optimize power production. Figure $2 \mathrm{~h}$ shows the pitch angle of blade 1 and Figure $2 \mathrm{i}$ shows the rotor torque. It is clearly visible that the proposed controller improves the tracking of the rated rotor speed while mitigating structural loads on the FOWT. Important points to note from the results presented in this section are

- The proposed controller improves dynamic response of the FOWT by reducing aerodynamic loads. Evidently, the improvement is greater in the along-wind direction since that is the objective of the LQ controller. But, as can be observed from Figure 2, there is reduction in the crosswind (direction perpendicular to inflow wind) structural responses arising from the reduction in aerodynamic loads.

- The LQ controller without integral action fails to track the rated rotor speed, Figure $2 \mathrm{~g}$, and rated generator power, Figure 2i. Operating the generator at above rated condition gives rise to undesirable loads on the generator.

- Improvements in platform pitch and roll is achieved by minimizing the oscillation corresponding to the platform pitch/roll frequency in the blade pitch angle. This is achieved by increasing the weight on the platform pitch degree of freedom in the LQ controller design. It can be observed from Figure 2e that using the proposed IPC the platform pitch DOF reaches steady-state faster than using the baseline controller. On the other hand, the proposed IPC increases the settling time of the platform roll DOF. However, it will be shown in the next section that under stochastic wind-wave condition including hydrodynamic damping the proposed IPC can improve the platform roll compared to the baseline controller.

- A 1P oscillation is introduced in the blade pitch angle, see Figure $2 \mathrm{~h}$, by the proposed controller that mitigates the $1 \mathrm{P}$ oscillation in the aerodynamic load and hence reduces the $1 \mathrm{P}$ oscillations of the blades, see Figure $2 \mathrm{a}$. Simultaneously, the integral controller ensures guaranteed tracking of the rated rotor speed by determining the low frequency variation of the pitch angle.

- It can be observed that the along-wind responses of the FOWT (blade out-of-plane displacement, tower fore-aft displacement and platform pitch rotation) reaches steadystate faster. For the cross-wind responses (blade inplane displacement, tower side-to-side displacement and platform roll rotation) the transient behaviour is not improved, however, the steady-state displacement/rotations are reduced.

- The improvement in structural response is obtained at an expense of increased pitch actuation, however, always within saturation limits.

To summarize, it can be noted that the proposed IPC improves the transient and steady-state behaviour of the FOWT by reducing the aerodynamic loads.

\section{Controller Performance Evaluation - STOCHASTIC WIND-WAVE}

The proposed controller is compared against the baseline controller with the FOWT subjected to stochastic wind-wave 


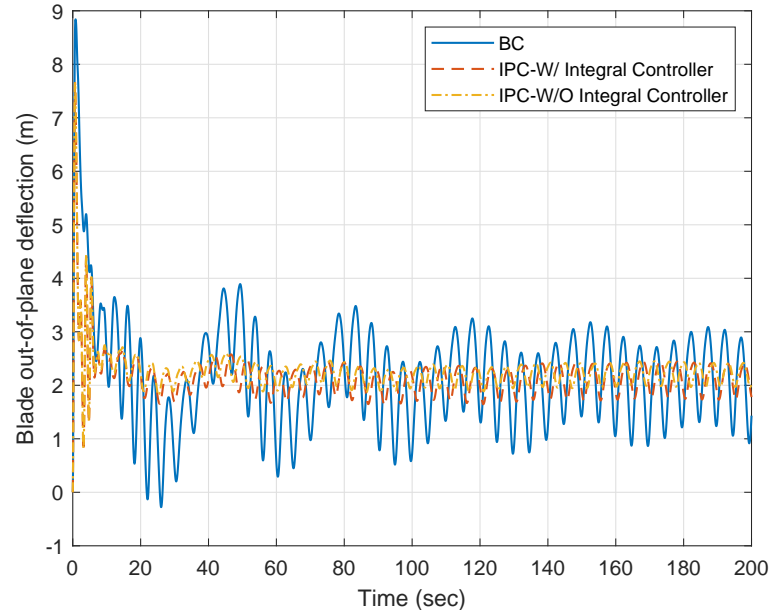

(a) Blade out-of-plane

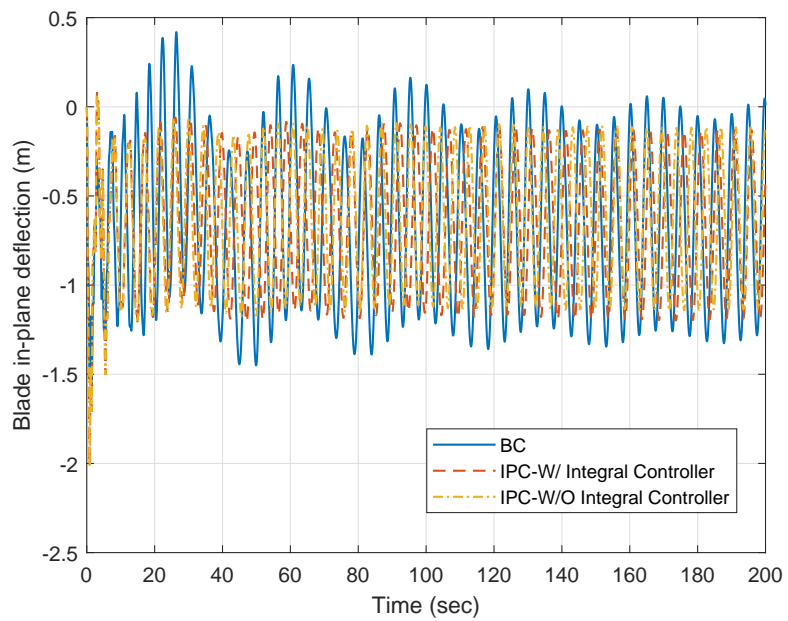

(b) Blade in-plane

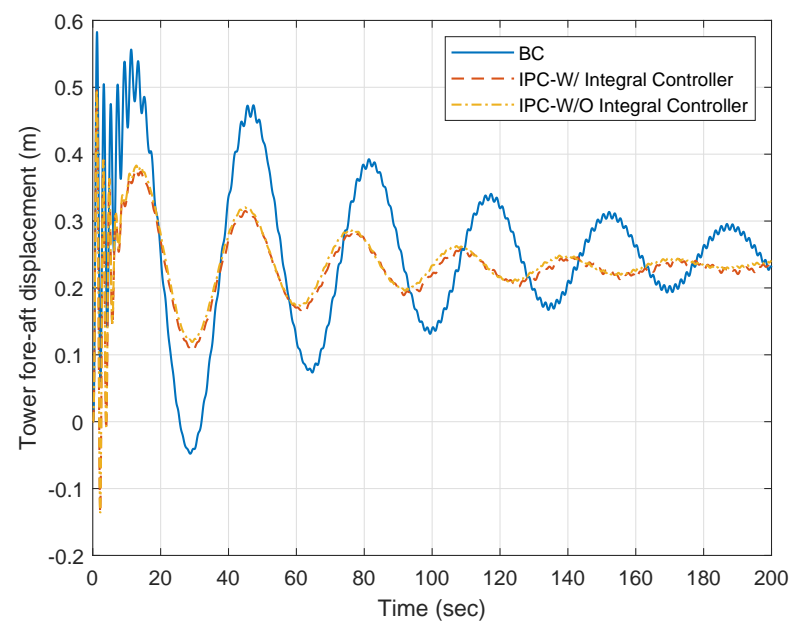

(c) Tower fore-aft

Fig. 2. FOWT subjected to steady $18 \mathrm{~m} / \mathrm{s}$ hub height wind speed

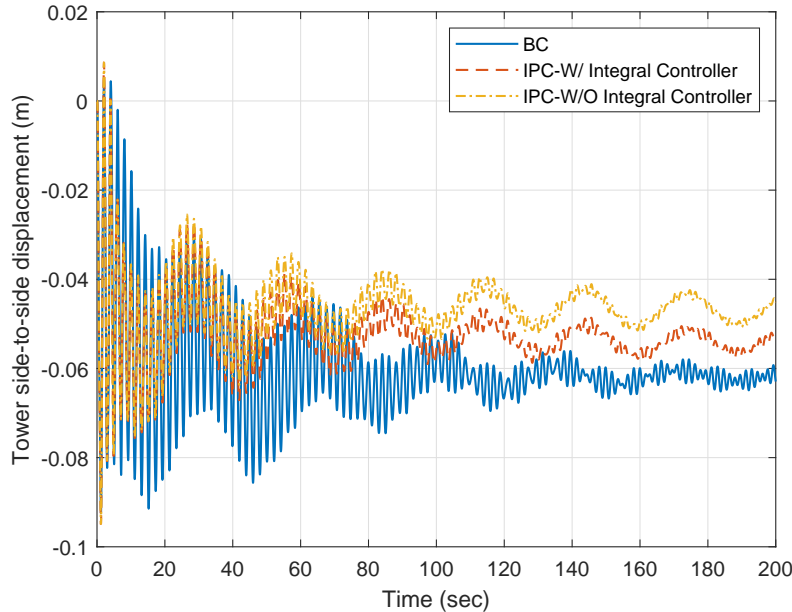

(d) Tower side-to-side

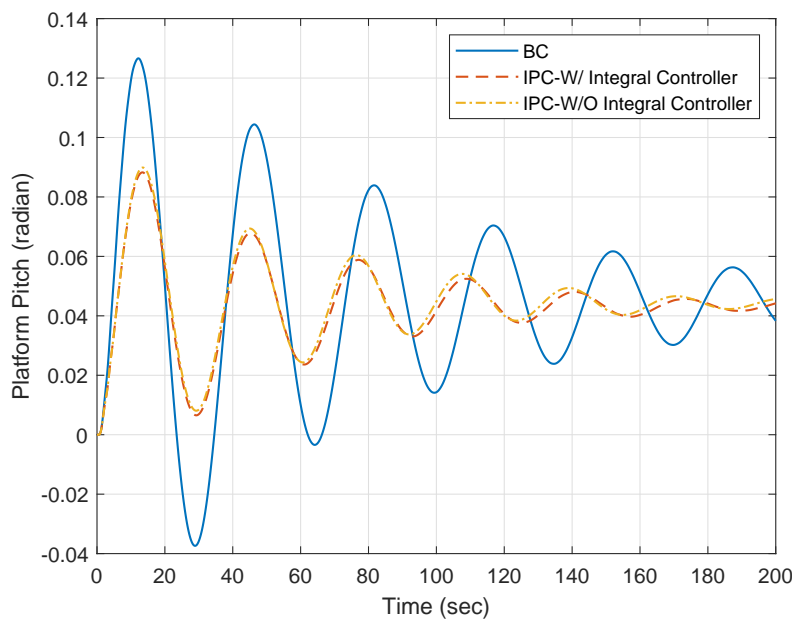

(e) Platform pitch

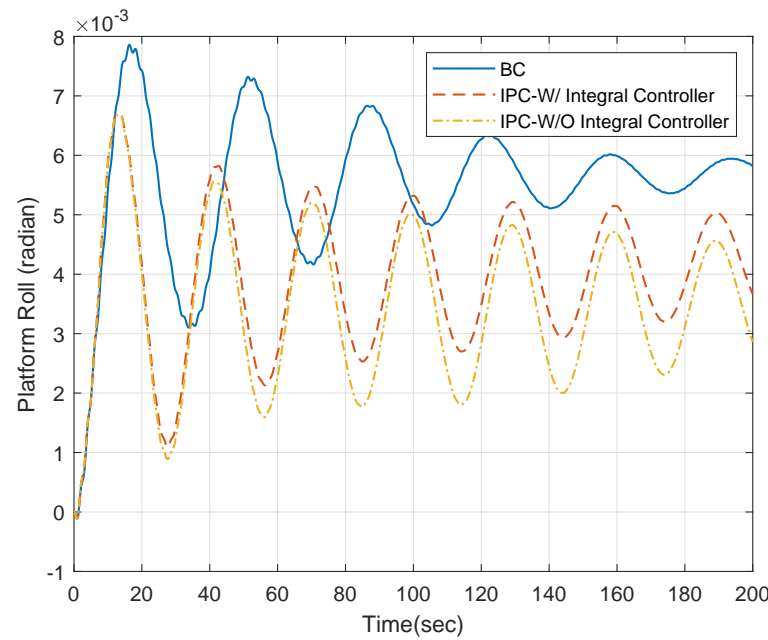

(f) Platform roll

Fig. 2. FOWT subjected to steady $18 \mathrm{~m} / \mathrm{s}$ hub height wind speed 


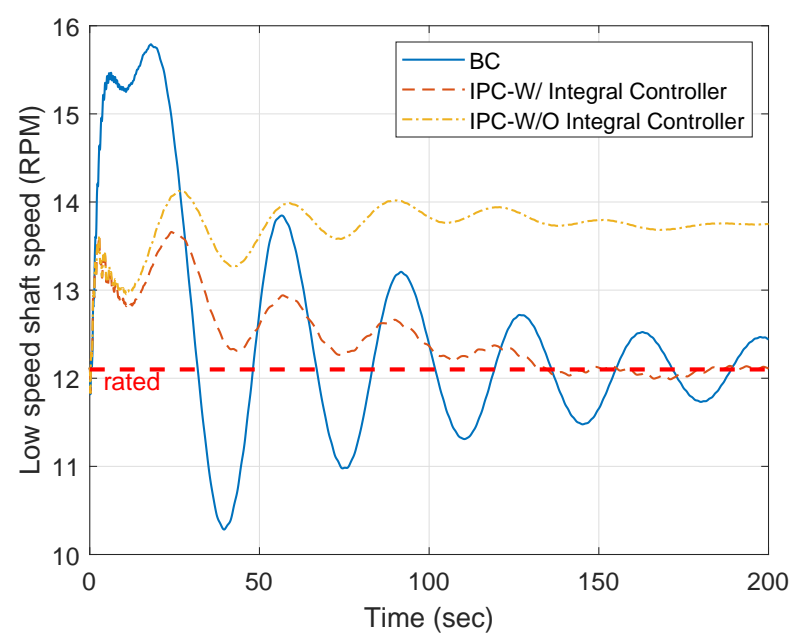

(g) Low speed shaft

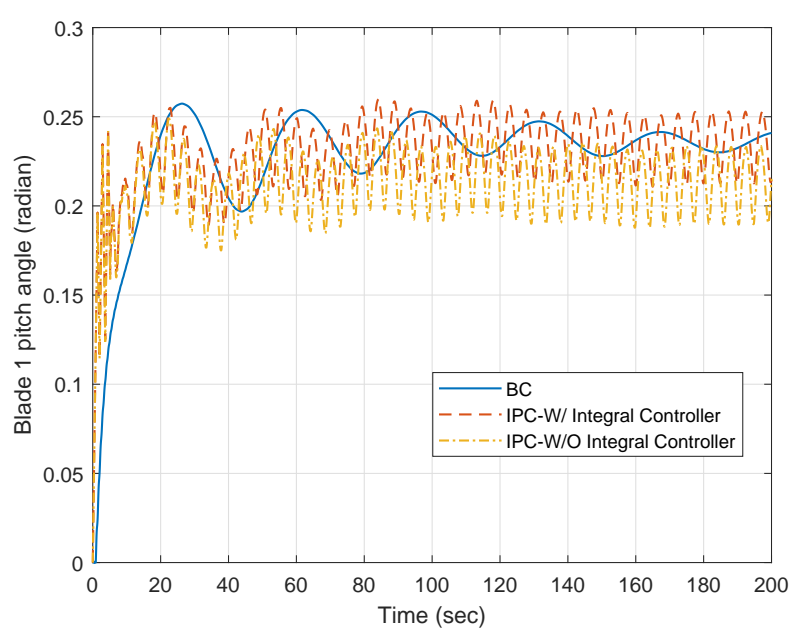

(h) Blade 1 pitch

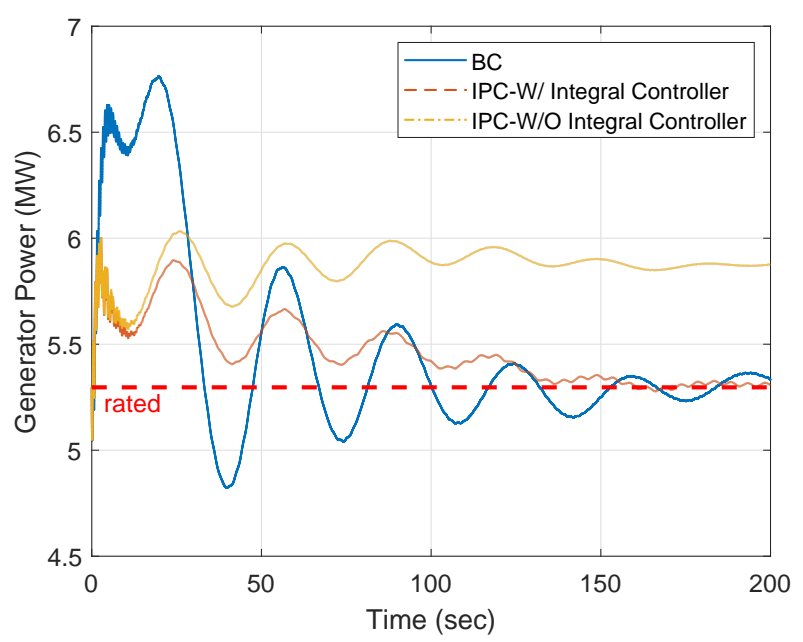

(i) Generator power

Fig. 2. FOWT subjected to steady $18 \mathrm{~m} / \mathrm{s}$ hub height wind speed loading environment. The turbulent wind field is generated using the TurbSim [29] package. The stochastic sea is modeled by the Pierson-Moskowitz spectrum [30]. Nine different windwave cases including cases with misaligned wind-wave loads are investigated in this paper. Wind-wave misalignment can often lead to increased vibrations in the cross-wind components of FOWT and hence the cases must be considered in the design of a controller. The turbulence intensity of the wind field is assumed to be $10 \%$ and the wave period for all different wave heights is assumed to be $6 \mathrm{~s}$. It is also assumed that the wave height increases with increasing wind speed. The selected load cases are summarized in Table I. It may be noted here that the selected load cases falls under design load case category 1.1 and 1.2 (normal and fatigue design load cases) of IEC 61400-1 $3^{\text {rd }}$ Ed. [31]. Cases 2, 4 and 6 simulate windwave misalignment and the wave direction relative to the wind direction is given in the table.

\section{A. Pareto Optimal Tuning}

In a multi-objective controller, the ratio between weights in the cost function balances the different control objectives. To find the right balance between the control objectives it is possible to sweep across the weight ratios to form Pareto fronts [32]. These Pareto fronts show the balance between the competitive objectives and can be used to tune the controller.

In this paper, optimal tuning parameters are determined by comparing the performance of the controller in terms of reduction of generator power variability $\left(\Psi_{P}\right)$ and the blade out-of-plane moment Damage Equivalent Load $\left(\Psi_{D E L}\right)$ defined in equation 9 .

$$
\begin{aligned}
\Psi_{P} & =1-\frac{\sigma_{I P C}}{\sigma_{B C}} \\
\Psi_{D E L} & =\frac{\Delta_{I P C}}{\Delta_{B C}}
\end{aligned}
$$

Where, $\sigma_{(\cdot)}$ is the generator power error RMS and $\Delta_{(\cdot)}$ is the fatigue DEL of the blade out-of-plane bending moment. To compute the Pareto curves the state weight matrix $\mathbf{Q}$ is modified as

$$
\mathbf{Q}=\left[\begin{array}{cc}
\mathbf{Q}_{l}(1-\rho) & \cdots \\
\cdots & \rho
\end{array}\right]
$$

where $\mathbf{Q}_{l}=0.001 \times \mathbf{I}_{11 \times 11} \cdot \mathbf{Q}_{l}$ is further modified by increasing the weight on the platform and tower degrees of freedom to 1 . $\mathbf{R}$ is left unchanged from the previous section. A sweep across the scalar tuning parameter $\rho$ is done to obtain the Pareto curves shown in Figure 3. Pareto curve for 3 different load cases are compared to determine the optimal tuning parameter $\rho$ for the entire range of wind speeds.

Optimal tuning is found by selecting a $\rho$ that gives maximum benefits in form of power regulation without a significant increase in cost in the form of fatigue DEL. In other words, it makes sense to increase the weight $\rho$ as long as the gradient of the Pareto curve is below a predefined small value. From visual inspection of the Pareto curves in Figure 3 the tuning $\rho$ has been set to 0.6 to achieve an optimal balance between the two competitive objectives. 


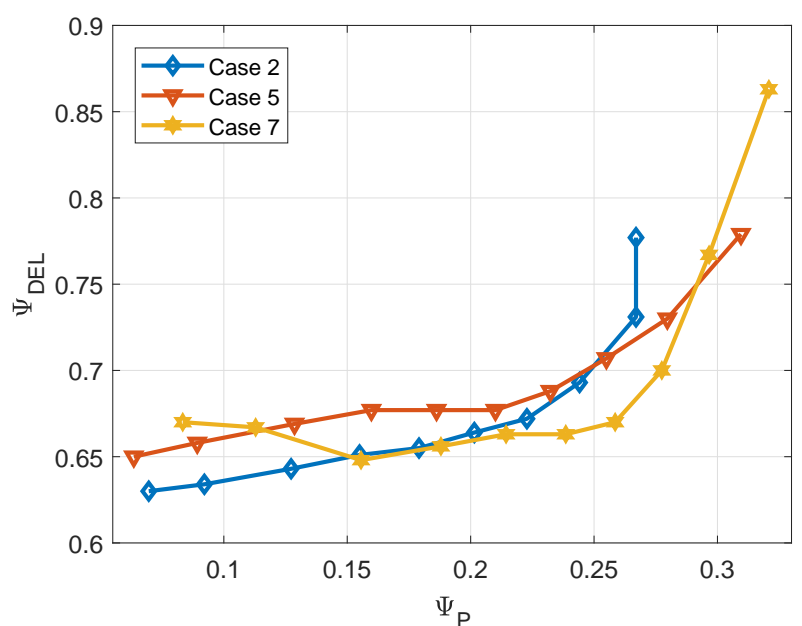

Fig. 3. Simulated Pareto curves

\section{B. Controller performance}

Figure 4 compares the fatigue DELs (Damage Equivalent Loads) of the blade out-of-plane bending moment, blade inplane bending moment, tower fore-aft bending moment and tower side-to-side bending moment of the FOWT obtained using the baseline controller with the proposed IPC. The DELs are estimated using the classical rainflow counting technique [33]. The DELs are obtained as the constant amplitude loads of fixed amplitude and a frequency of $1 \mathrm{~Hz}$. The first $50 \mathrm{~s}$ of the time history load predictions are removed from the analysis to cater to transient effects. The figures clearly show that significant reduction in fatigue DELs can be obtained from the proposed IPC.

Next, the standard deviations of the platform pitch and roll rotations are shown in Figure 5. It can be observed that the proposed IPC significantly reduces platform pitch rotation for every different load case investigated here. It is also important to note that the platform roll rotation is also reduced by the proposed IPC.

It is of significant interest to note here that the RMS (root mean square) of the low speed shaft error and generator power error is reduced consistently, (see Figure 6a and Figure $6 \mathrm{~b}$ respectively) over the different load cases. This shows that the proposed IPC is better than the baseline controller in regulating power production of the FOWT.

The wave loads effects the dynamic response of the tower most significantly. It can be observed from Figure $4 \mathrm{c}$ and Figure $4 \mathrm{~d}$ that increasing wave height increases the tower base fore-aft and side-to-side moments. It can be observed from load cases 2, 4 and 6 that the wind-wave miss-alignment significantly increases the tower side-to-side moment and decreases tower fore-aft moment. However, the proposed controller performs better than the baseline controller in achieving the design objectives for the wind-wave misalignment cases. Although wave loads influences the dynamics of the entire FOWT its effect on the other degrees of freedom is not apparently visible from the results presented here because wind is the primary source of dynamic loads on a spar-type
TABLE I

LOAD CASES

\begin{tabular}{|c|c|c|c|c|}
\hline $\begin{array}{c}\text { Case } \\
\text { No. }\end{array}$ & $\begin{array}{c}\text { Rotor Speed } \\
(\mathrm{rpm})\end{array}$ & $\begin{array}{c}\text { Wind Speed } \\
v(\mathrm{~m} / \mathrm{s})\end{array}$ & $\begin{array}{c}\text { Wave Height } \\
H_{s}(\mathrm{~m})\end{array}$ & $\begin{array}{c}\text { Wave Direction } \\
\text { (degrees) }\end{array}$ \\
\hline 1 & 12.1 & 13 & 1 & 0 \\
2 & 12.1 & 13 & 2 & 30 \\
3 & 12.1 & 15 & 2 & 0 \\
4 & 12.1 & 15 & 2 & 60 \\
5 & 12.1 & 17 & 3 & 0 \\
6 & 12.1 & 17 & 2 & 90 \\
7 & 12.1 & 19 & 4 & 0 \\
8 & 12.1 & 21 & 5 & 0 \\
9 & 12.1 & 23 & 6 & 0 \\
\hline
\end{tabular}

TABLE II

DLC 1.2 AVERage Fatigue DELS

\begin{tabular}{|c|c|c|c|c|}
\hline Controller & $\begin{array}{c}\text { Blade } \\
\text { out-of-plane } \\
\text { moment } \\
(\mathrm{kNm})\end{array}$ & $\begin{array}{c}\text { Blade } \\
\text { in-plane } \\
\text { moment } \\
(\mathrm{kNm})\end{array}$ & $\begin{array}{c}\text { Tower } \\
\text { fore-aft } \\
\text { moment } \\
(\mathrm{kNm})\end{array}$ & $\begin{array}{c}\text { Tower } \\
\text { side-to-side } \\
\text { moment } \\
(\mathrm{kNm})\end{array}$ \\
\hline BC & $2.44 \mathrm{E}+003$ & $2.25 \mathrm{E}+003$ & $1.98 \mathrm{E}+004$ & $1.20 \mathrm{E}+004$ \\
IPC & $1.50 \mathrm{E}+003$ & $2.13 \mathrm{E}+003$ & $1.40 \mathrm{E}+004$ & $9.70 \mathrm{E}+003$ \\
\hline
\end{tabular}

\section{FOWT.}

To summarize, the best case, worst case and average reduction in fatigue DELs, standard deviations of the platform rotations and the RMS of the rotor speed error and generator power error achieved by the propose IPC over the baseline controller is presented in Table III.

The improvements in the form of reduced loads and improved power regulation is obtained at a cost of higher pitch actuation. To evaluate this increase an equivalent constant amplitude blade pitch cycle is estimated from the time history predictions of the blade pitch angles using the rainflow counting method. The resulting pitch cycle has a constant amplitude and a frequency of $1 \mathrm{~Hz}$. The constant amplitude obtained from the baseline controller in compared against the proposed IPC and presented in Figure 7. It can be clearly observed that pitch actuation is higher compared to the baseline controller for all load cases. Also, using the baseline controller the blade pitch actuation reduces with wind speed, however, using the proposed IPC blade pitch actuation increases with wind speed. Although this increase may not be ideal in all situations, with reliable pitch systems the control strategy proposed in this paper is capable of greatly improving FOWT response and power production.

\section{Comparison against published literature}

In this subsection, the proposed IPC is compared against the most popular IPCs available in the literature. For reasonable comparison, it is important to compare the performance of the controllers on the same 5MW OC3 Hywind spar-type offshore wind turbine [27].

Namik and Stol [19] proposed two IPCs, one based on an SFC control strategy and another based on a DAC control strategy. The authors compared the proposed controller against 


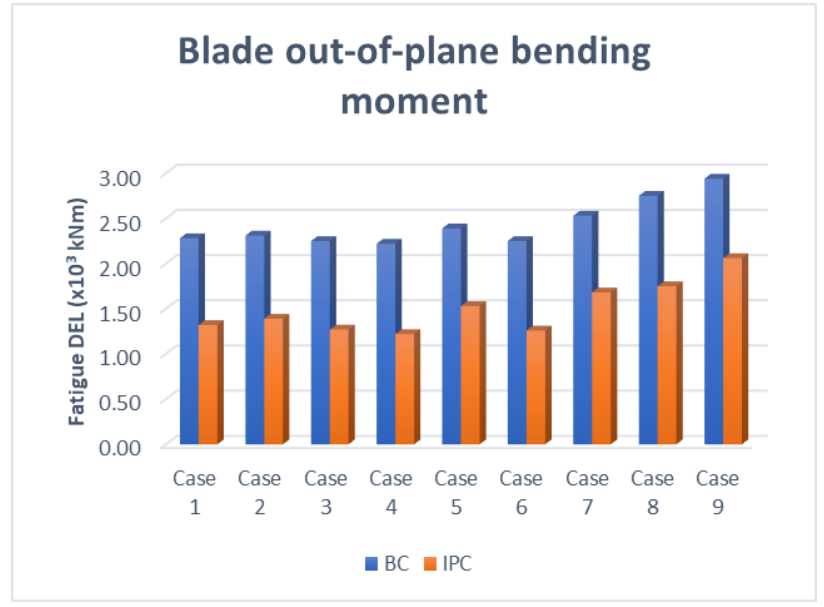

(a)

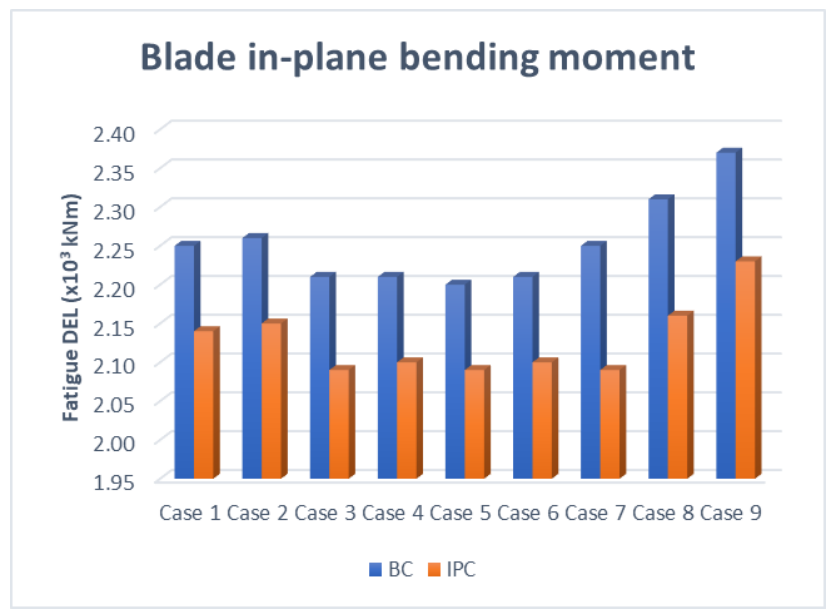

(b)

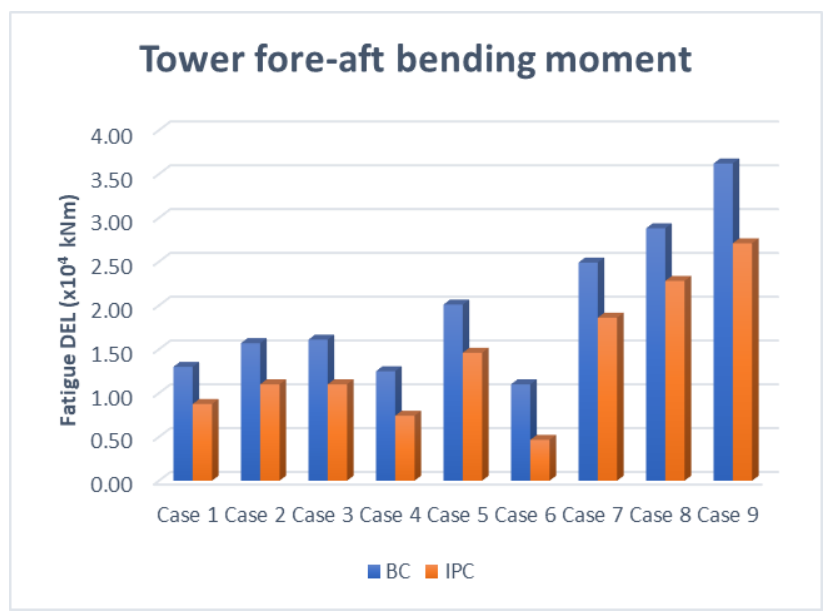

(c)

Fig. 4. Fatigue DELs

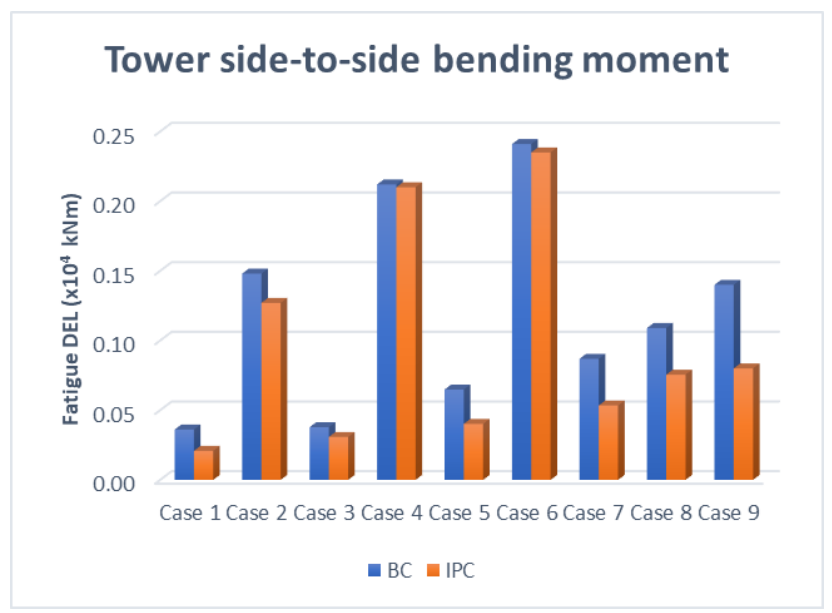

(d)

Fig. 4. Fatigue DELs



(a)

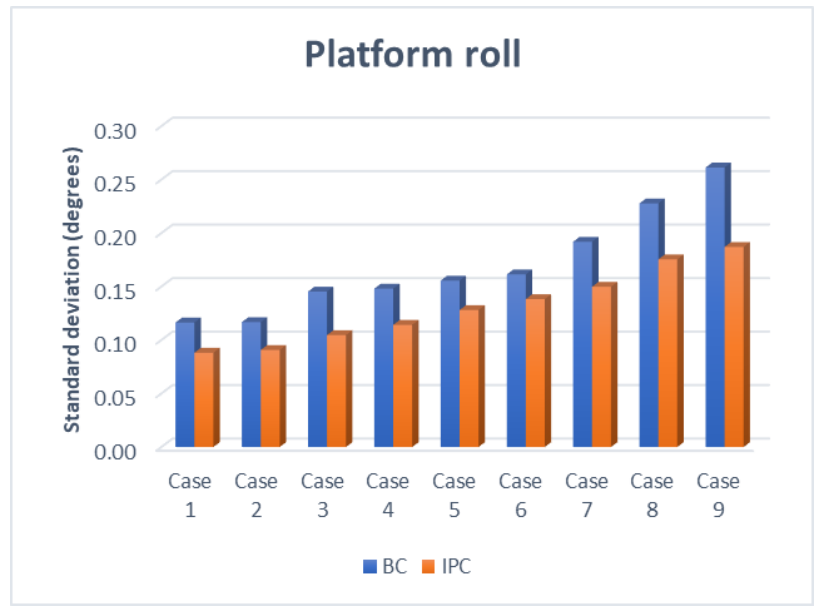

(b)

Fig. 5. Standard deviations of platform responses 


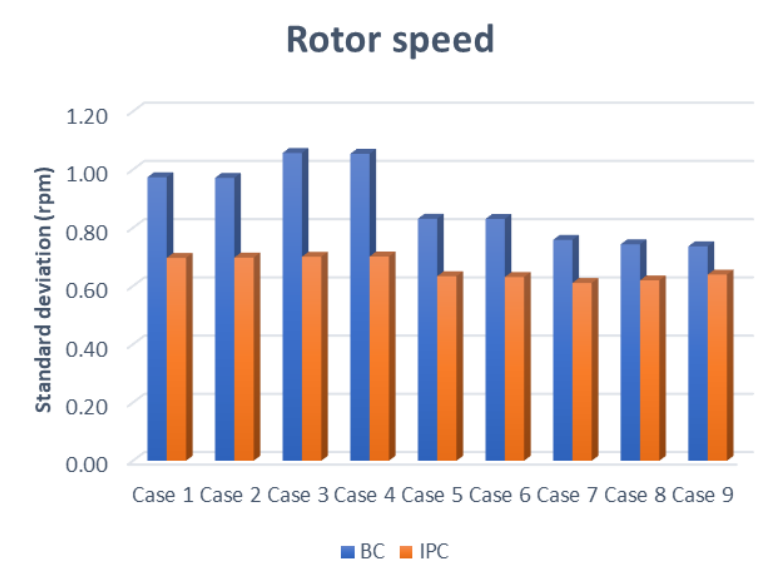

(a)

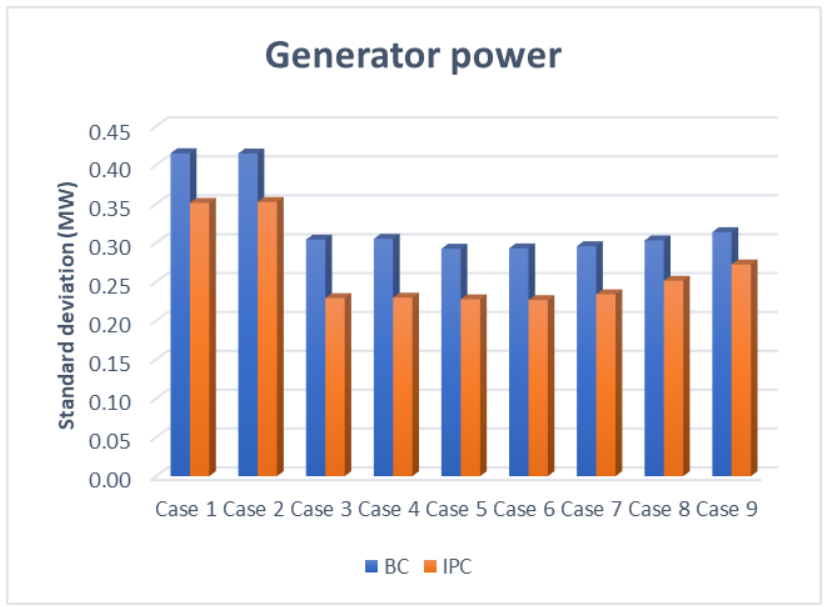

(b)

Fig. 6. RMS of rotor speed and generator power error

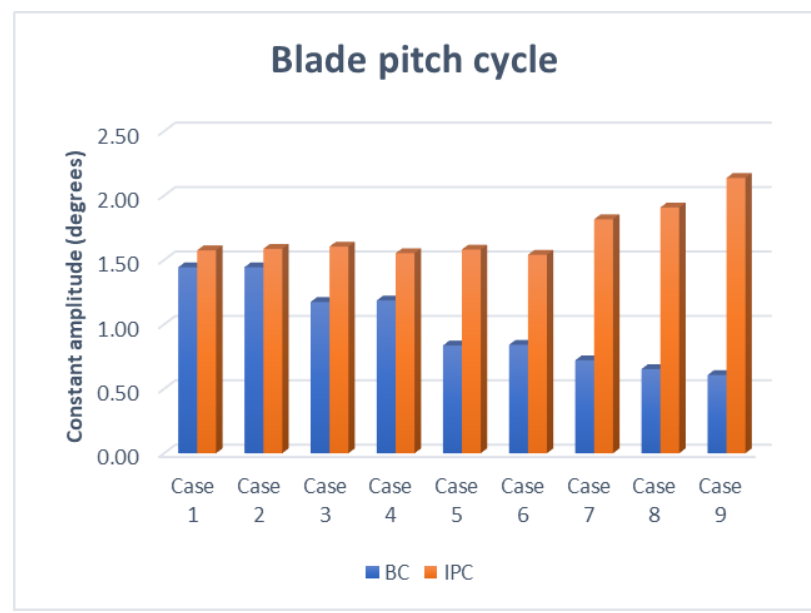

Fig. 7. Equivalent constant amplitude pitch cycle
TABLE III

PERCENTAGE REDUCTION IN STANDARD DEVIATION OVER BASELINE CONTROLLER

\begin{tabular}{|c|c|c|c|c|}
\hline \multicolumn{2}{|c|}{ Fatigue DELs and responses } & $\begin{array}{l}\text { Best } \\
\text { case }\end{array}$ & $\begin{array}{l}\text { Worst } \\
\text { case }\end{array}$ & Average \\
\hline \multirow{2}{*}{$\begin{array}{l}\text { Platform } \\
\text { RMS }\end{array}$} & Pitch & 66 & 44 & 58 \\
\hline & Roll & 28 & 14 & 23 \\
\hline \multirow{4}{*}{$\begin{array}{l}\text { Fatigue } \\
\text { DELs }\end{array}$} & $\begin{array}{c}\text { Tower fore aft } \\
\text { bending moment }\end{array}$ & 58 & 21 & 32 \\
\hline & $\begin{array}{l}\text { Tower side to } \\
\text { side bending } \\
\text { moment }\end{array}$ & 43 & 1 & 25 \\
\hline & $\begin{array}{l}\text { Blade out of } \\
\text { plane bending } \\
\text { moment }\end{array}$ & 45 & 30 & 39 \\
\hline & $\begin{array}{l}\text { Blade in plane } \\
\text { bending moment }\end{array}$ & 7 & 5 & 6 \\
\hline \multirow{2}{*}{$\begin{array}{l}\text { Generator } \\
\text { error RMS }\end{array}$} & Rotor speed & 34 & 13 & 25 \\
\hline & Generator power & 25 & 13 & 20 \\
\hline
\end{tabular}

the baseline controller [7]. They reported $9 \%$ reduction in tower fatigue DELs over the baseline controller. A massive $64 \%$ reduction in power error has also been reported. However, as the authors have mentioned in their paper this is due to the use of constant power algorithm used by the generator torque controller and not due to the proposed IPCs. The authors do not report any reduction in the blade fatigue DELs or any improvement in the floating platform response. In comparison, the controller proposed in this paper achieves greater reductions in fatigue DELs of the blades and the tower. It significantly improves the floating platform response and also improves generator power output using a control torque algorithm which is recommended by [7]. The reductions are provided in Table III. It can be observed that the proposed controller performs significantly better than the one proposed in [19].

In another paper, Raach et al. [10] proposed an IPC based on a nonlinear model predictive control strategy. The proposed controller was compared against the baseline controller [7]. They have reported around 18\% reduction in blade out-ofplane fatigue DELs and an impressive $77.6 \%$ reduction in generated power error. In comparison, the controller proposed in this paper is better in reducing fatigue DELs although poorer in reducing generator power error. However, this comparison is not reasonable as the NMPC controller requires $1.3 \mathrm{~s}$ for solving the optimization problem to determine the control input. This considerable time delay will significantly affect the performance of the controller which has not been considered by the authors. Moreover, the authors consider only one load case and the performance of the controller over the operational region 3 has not been investigated.

\section{CONCLUSION}

A new individual blade pitch control strategy has been proposed in this paper that combines a low authority LQ controller with an integral controller to achieve improved tracking of the rated rotor speed to optimze power regulation while 
simultaneously reducing aerodynamic loads on the FOWT. The proposed controller is compared against the baseline controller used by FAST [7] with the FOWT subjected to various stochastic wind-wave scenarios. It has been shown numerically that the proposed controller performs better than the existing baseline controller and other existing controllers in the literature. The important conclusions to draw from this study are

- The proposed controller is more effective compared to the baseline controller in reducing the variability in the rotor speed thus improving the power production of the FOWT. Unlike the IPCs available in literature, the proposed controller is capable of simultaneously mitigating FOWT structural responses and rotor power variability.

- The simple and intuitive nature of the controller makes it easy to design. The successful use of a reduced order model of the FOWT and a linearized approximation of the control influence matrix shows that exact information of the fully non-linear wind turbine model is not required to design an effective controller.

- The proposed controller is equally effective for cases with wind-wave misalignment.

- Significant reduction in blade vibrations and platform motion is observed. Excessive blade vibrations and platform motion are critical for functionality of the wind turbine. Hence, the reductions achieved by the proposed controller can significantly improve the performance of the FOWT.

- As shown in this paper, this improvement in performance, notably the reduction of $1 \mathrm{P}$ oscillation of the aerodynamic loads is achieved at an expense of increase blade pitch actuation, especially at higher wind speeds. However, this is always well within saturation limits.

\section{APPENDIX A \\ DEGREES OF FREEDOM OF THE COMPLETE NON-LINEAR MODEL}

The complete non-linear floating offshore wind turbine model has 22 degrees of freedom. Six degrees of freedom have been used to describe the motion of the platform. Four degrees of freedom have been used to describe the motion of the tower. One degree of freedom has been used to describe the rotation of the nacelle. The rotation of the low speed shaft has been modelled using two degrees of freedom. And finally, nine degrees of freedom have been used to describe the motion of the three blades. The degrees of freedom are listed in Table IV.

\section{APPENDIX B}

\section{EQUATIONS OF MOTION OF THE REDUCED ORDER MODEL}

The linearized equations of motion of the 6 degree of freedom system is described in this section. To derive the equations of motion first it is assumed that the wind turbine is rotating at a constant speed. Hence, the generator speed error is basically zero and it is not a degree of freedom. The equations of motion of the 5 degree of freedom system is then augmented by the generator speed error degree of freedom to obtain the final 6 degree of freedom system. Understandably,
TABLE IV

DEGREES OF FREEDOM

$\begin{array}{ll}q_{S g} & \text { Platform surge } \\ q_{S w} & \text { Platform sway } \\ q_{H v} & \text { Platform heave } \\ q_{R} & \text { Platform roll } \\ q_{P} & \text { Platform pitch } \\ q_{Y} & \text { Platform yaw } \\ q_{T F A 1} & \text { First tower fore-aft bending mode } \\ q_{T F A 2} & \text { Second tower fore-aft bending mode } \\ q_{T S S 1} & \text { First tower side-to-side bending mode } \\ q_{T S S 2} & \text { Second tower side-to-side bending mode } \\ q_{y a w} & \text { Nacelle yaw } \\ q_{G e A z} & \text { Generator azimuth angle } \\ q_{D r T r} & \text { Drive-train torsional flexibility } \\ q_{B i F 1} & \text { First flapwise bending mode for } i^{t h} \text { blade } \\ q_{B i F 2} & \text { Second flapwise bending mode for } i^{t h} \text { blade } \\ q_{B i E 1} & \text { First edgewise bending mode for } i^{t h} \text { blade }\end{array}$

the generator speed error degree of freedom is decoupled from the rest of the system. This approach is undertaken since the inclusion of the generator speed error as a degree of freedom introduces non-linearities in the form of cosine and sine terms which cannot be linearized using small angle approximation. We first look at the generator speed error degree of freedom

$$
\begin{aligned}
& \dot{q}_{\varepsilon}=\delta \Omega=\Omega_{0}-q_{G e A z} \\
& q_{\varepsilon}=\int_{0}^{t} \dot{q}_{\varepsilon} d t=\int_{0}^{t}\left(\Omega_{0}-q_{G e A z}\right) d t \\
& \ddot{q}_{\varepsilon}=\delta \dot{\Omega}=\ddot{q}_{G e A z}
\end{aligned}
$$

Where, $\Omega_{0}$ is the rated rated rotor speed of the FOWT. The equation of motion of this degree of freedom assuming only intergal action $K_{I}$ can be obtained from [24] as

$$
I_{D T} \ddot{q}_{\varepsilon}+\left(-\frac{P_{0}}{\Omega_{0}^{2}}\right) \dot{q}_{\varepsilon}+\frac{1}{\Omega_{0}}\left(-\frac{\partial P_{0}}{\partial \theta}\right) N_{G e a r} K_{I} q_{\varepsilon}=0
$$

Where, $I_{D T}=I_{\text {Rotor }}+N_{\text {Gear }}^{2} I_{G e n}$ is the drive-train intertia cast into the low speed shaft, $I_{\text {Rotor }}$ is the inertia of the rotor, $I_{G e n}$ is the inertia of the generator relative to the high speed shaft, $N_{\text {Gear }}$ is the gear box ratio. $P_{0}$ is the rated mechanical power. As recommended by [27] the integral gain $K_{I}$ is selected such that the frequency of this degree of freedom is $0.2 \mathrm{rad} / \mathrm{s}$. Therefore, the stiffness term can be written as

$$
\frac{1}{\Omega_{0}}\left(-\frac{\partial P_{0}}{\partial \theta}\right) N_{G e a r} K_{I}=0.2^{2} I_{D T}=k_{\varepsilon \varepsilon}
$$

The mass, stiffness and damping matrix of the linearized six degree of freedom system are obtained as

$$
\begin{aligned}
& \mathbf{M}=\left[\begin{array}{cccccc}
m_{P P} & m_{P T} & m_{P B 1} & m_{P B 2} & m_{P B 3} & 0 \\
& m_{T T} & m_{T B 1} & m_{T B 2} & m_{T B 3} & 0 \\
& & m_{B B} & 0 & 0 & 0 \\
& & & m_{B B} & 0 & 0 \\
& & & & m_{B B} & 0 \\
& & & & 0 & I_{D T}
\end{array}\right] \\
& \mathbf{C}=\left[\begin{array}{cccccc}
c_{P P} & 0 & 0 & 0 & 0 & 0 \\
& c_{T T} & 0 & 0 & 0 & 0 \\
& & c_{B B} & 0 & 0 & 0 \\
& & & c_{B B} & 0 & 0 \\
& \text { sym } & & & c_{B B} & 0 \\
& & & & & \left(-\frac{P_{0}}{\Omega_{0}^{2}}\right)
\end{array}\right]
\end{aligned}
$$




$$
\mathbf{K}=\left[\begin{array}{cccccc}
k_{P P} & 0 & 0 & 0 & 0 & 0 \\
& k_{T T} & 0 & 0 & 0 & 0 \\
& & k_{B B}^{1} & 0 & 0 & 0 \\
& & & k_{B B}^{2} & 0 & 0 \\
& \text { sym } & & & k_{B B}^{3} & 0 \\
& & & & 0 & k_{\varepsilon \varepsilon}
\end{array}\right]
$$

where sym stands for symmetric and denotes that the above matrices are symmetric. The quantities in the above matrices can be given as

$$
\begin{aligned}
m_{P P}= & 12 H_{t}^{2} \int_{0}^{L} m_{b}(r) \mathrm{d} r+\sum_{i=1}^{3}\left[4 H_{t} \int_{0}^{L} r m_{b}(r) \mathrm{d} r \cos \left(\psi_{i}\right)\right. \\
& \left.+\int_{0}^{L} r^{2} m_{b}(r) \mathrm{d} r \cos ^{2}\left(\psi_{i}\right)\right]+\int_{0}^{H_{t}} h m_{t}(h) \mathrm{d} h \\
+ & H_{t}^{2} m_{N H}+I_{P}+I_{P}^{A M} \\
m_{P T} & =6 H_{t} \int_{0}^{L} m_{b}(r) \mathrm{d} r+\int_{0}^{H_{t}} h m_{t}(h) \phi_{t}(h) \mathrm{d} h \\
m_{P B i} & =2 H_{t} \int_{0}^{L} \phi_{b}(r) m_{b}(r) \mathrm{d} r \\
& +\int_{0}^{L} r \phi_{b}(r) m_{b}(r) \mathrm{d} r \cos \left(\psi_{i}\right) \\
m_{T T} & =3 \int_{0}^{L} m_{b}(r) \mathrm{d} r+\int_{0}^{H_{t}} \phi_{t}^{2} m_{t}(h) \mathrm{d} h+m_{N H}
\end{aligned}
$$

Where, $H_{t}$ is the height of the tower and $L$ is the length of the blades. $m_{b}(r)$ and $m_{t}(h)$ are mass per unit length of the blades and tower respectively. $\psi_{i}$ is the azimuth angle of the $i^{\text {th }}$ blade. $m_{N H}$ is the combined mass of the nacelle and hub. $I_{P}$ is the rotational inertia of the platform and $I_{P}^{A M}$ is the hydrodynamic added mass coefficient associated with the platform pitch degree of freedom. $\phi_{b}(r)$ and $\phi_{t}(h)$ are the normalized fundamental model shapes of the blades and tower respectively. $c_{P P}$ is the linear hydrodynamic damping coefficient associated with the platform pitch degree of freedom, $c_{T T}$ and $c_{B B}$ are structural damping coefficient of the tower and the blades respectively. $k_{P P}$ is the summation of hydro-static and mooring lines stiffness associated with the platform pitch degree of freedom. $k_{T T}$ is the elastic stiffness of the tower and $k_{B B}^{i}=k_{B B}^{e}+k_{B B}^{c}+k_{B B}^{g} \cos \left(\psi_{i}\right)$ is the summation of the elastic stiffness $k_{B B}^{e}$ centrifugal stiffness $k_{B B}^{c}$ and gravitational stiffening/softening of the $i^{\text {th }}$ blade. More details on these terms can be found in [34]-[36].

\section{REFERENCES}

[1] E. Bossanyi, "Individual blade pitch control for load reduction," Wind energy, vol. 6, no. 2, pp. 119-128, 2003.

[2] —_ "Further load reductions with individual pitch control," Wind energy, vol. 8, no. 4, pp. 481-485, 2005.

[3] E. A. Bossanyi, P. A. Fleming, and A. D. Wright, "Validation of individual pitch control by field tests on two-and three-bladed wind turbines," IEEE Transactions on Control Systems Technology, vol. 21, no. 4, pp. 1067-1078, 2013.
[4] M. H. Mughal and L. Guojie, "Review of pitch control for variable speed wind turbine," in 2015 IEEE 12th Intl Conf on Ubiquitous Intelligence and Computing and 2015 IEEE 12th Intl Conf on Autonomic and Trusted Computing and 2015 IEEE 15th Intl Conf on Scalable Computing and Communications and Its Associated Workshops (UIC-ATC-ScalCom). IEEE, 2015, pp. 738-744.

[5] S. Schuler, D. Schlipf, P. W. Cheng, and F. Allgöwer, " $\ell_{1}$-optimal control of large wind turbines," IEEE Transactions on Control Systems Technology, vol. 21, no. 4, pp. 1079-1089, 2013.

[6] H. Jafarnejadsani and J. Pieper, "Gain-scheduled $\ell_{1}$-optimal control of variable-speed-variable-pitch wind turbines," IEEE Transactions on Control Systems Technology, vol. 23, no. 1, pp. 372-379, 2015.

[7] J. M. Jonkman and M. L. Buhl Jr, "Fast user's guide-updated august 2005," National Renewable Energy Laboratory (NREL), Golden, CO. Tech. Rep., 2005.

[8] J. Cheon, S. Kwon, and Y. Choi, "Design of a pitch controller using disturbance accommodating control for wind turbines under stochastic environments," in Industrial Electronics (ISIE), 2014 IEEE 23rd International Symposium on. IEEE, 2014, pp. 2572-2577.

[9] D. Schlipf, D. J. Schlipf, and M. Kühn, "Nonlinear model predictive control of wind turbines using lidar," Wind energy, vol. 16, no. 7, pp. 1107-1129, 2013.

[10] S. Raach, D. Schlipf, F. Sandner, D. Matha, and P. W. Cheng, "Nonlinear model predictive control of floating wind turbines with individual pitch control," in 2014 American Control Conference. IEEE, 2014, pp. 44344439.

[11] V. Petrović, M. Jelavić, and M. Baotić, "Advanced control algorithms for reduction of wind turbine structural loads," Renewable Energy, vol. 76, pp. 418-431, 2015.

[12] R. Gao and Z. Gao, "Pitch control for wind turbine systems using optimization, estimation and compensation," Renewable Energy, vol. 91, pp. 501-515, 2016.

[13] Y. Ren, L. Li, J. Brindley, and L. Jiang, "Nonlinear PI control for variable pitch wind turbine," Control Engineering Practice, vol. 50, pp. 84-94, 2016.

[14] Y. Yuan and J. Tang, "Adaptive pitch control of wind turbine for load mitigation under structural uncertainties," Renewable Energy, vol. 105, pp. 483-494, 2017.

[15] J. Lan, R. J. Patton, and X. Zhu, "Fault-tolerant wind turbine pitch control using adaptive sliding mode estimation," Renewable Energy, vol. 116, pp. 219-231, 2018.

[16] B. Fitzgerald, A. Staino, and B. Basu, "Wavelet-based individual blade pitch control for vibration control of wind turbine blades," Structural Control and Health Monitoring, vol. 26, no. 1, p. e2284, 2019.

[17] H. Namik and K. Stol, "Individual blade pitch control of floating offshore wind turbines," Wind Energy: An International Journal for Progress and Applications in Wind Power Conversion Technology, vol. 13, no. 1, pp. 74-85, 2010.

[18] — "Performance analysis of individual blade pitch control of offshore wind turbines on two floating platforms," Mechatronics, vol. 21, no. 4, pp. 691-703, 2011.

[19] 1 , "Individual blade pitch control of a spar-buoy floating wind turbine," system, vol. 1, no. 3, p. 6, 2014.

[20] A. Staino and B. Basu, "Emerging trends in vibration control of wind turbines: a focus on a dual control strategy," Philosophical Transactions of the Royal Society A: Mathematical, Physical and Engineering Sciences, vol. 373, no. 2035, p. 20140069, 2015.

[21] S. Sarkar and B. Fitzgerald, "Vibration control of spar-type floating offshorewind turbine towers using a tuned mass-damper-inerter," Structural Control and Health Monitoring, p. e2471, 2019.

[22] M. Hall, "Moordynusers guide," Department of Mechanical Engineering, University of Maine: Orono, ME, USA, 2015.

[23] S. Sarkar, B. Fitzgerald, and B. Basu, "Development of a flexible multibody dynamics wind turbine model following Kane's method," in Proceedings of the Civil Engineering Research in Ireland, 2018, pp. 883-888.

[24] J. M. Jonkman, Dynamics modeling and loads analysis of an offshore floating wind turbine. University of Colorado at Boulder, 2007.

[25] T. R. Kane and D. A. Levinson, Dynamics, theory and applications. McGraw Hill, 1985.

[26] J. M. Jonkman, S. Butterfield, W. Musial, and G. Scott, Definition of a 5-MW reference wind turbine for offshore system development. National Renewable Energy Laboratory Golden, CO, 2009.

[27] J. M. Jonkman, Definition of the Floating System for Phase IV of OC3. Citeseer, 2010.

[28] MATLAB, version 9.4.0 (R2018a). Natick, Massachusetts: The MathWorks Inc., 2018. 
[29] B. J. Jonkman, “Turbsim user's guide: Version 1.50," National Renewable Energy Lab.(NREL), Golden, CO (United States), Tech. Rep., 2009.

[30] W. J. Pierson Jr and L. Moskowitz, "A proposed spectral form for fully developed wind seas based on the similarity theory of sa kitaigorodskii," Journal of geophysical research, vol. 69, no. 24, pp. 5181-5190, 1964.

[31] I. E. Commission et al., "Iec 61400-1: Wind turbines part 1: Design requirements," International Electrotechnical Commission, 2005.

[32] P. F. Odgaard, L. F. Larsen, R. Wisniewski, and T. G. Hovgaard, "On using pareto optimality to tune a linear model predictive controller for wind turbines," Renewable Energy, vol. 87, pp. 884-891, 2016.

[33] S. D. Downing and D. Socie, "Simple rainflow counting algorithms," International journal of fatigue, vol. 4, no. 1, pp. 31-40, 1982.

[34] B. Fitzgerald, B. Basu, and S. R. Nielsen, "Active tuned mass dampers for control of in-plane vibrations of wind turbine blades," Structural Control and Health Monitoring, vol. 20, no. 12, pp. 1377-1396, 2013.

[35] S. Sarkar and A. Chakraborty, "Optimal design of semiactive mr-tlcd for along-wind vibration control of horizontal axis wind turbine tower," Structural Control and Health Monitoring, vol. 25, no. 2, 2018.

[36] B. Fitzgerald, S. Sarkar, and A. Staino, "Improved reliability of wind turbine towers with active tuned mass dampers (atmds)," Journal of Sound and Vibration, vol. 419, pp. 103-122, 2018. 